\title{
Multi cluster optimization using adaptive energy constraint in wireless sensor network
}

\author{
${ }^{1}$ Kothakapu Chandra Reddy, ${ }^{2} \mathbf{G}$. Yugandhar \\ Department of Computer Science and Engineering, GITAM Deemed to be University, Hyderabad
}

\begin{abstract}
Energy constraint is a major concern in the deployment and accessing of wireless sensor network. Due to remote usage and constant power supply, operation of the node is highly dependent on the approach of energy conservation used. The advantage of wireless sensor network (WSN) in practical usage has brought out the need of utilizing this network in wide area usage. in a large distributed network, communication with energy conservation could be achieved with multi cluster communication. Cluster based communication in WSN is proved to be a efficient approach of energy conservation, where the communication are developed using cluster Head and cluster member nodes. The effort on optimal head selection, and deployment in a energy conservation for multi cluster approach were developed in recent works. Wherein effort is made in optimal selection of head, cluster members are registered based on the range constraint. The random addition of cluster member has a limitation over energy utilization and interference model. In this paper, an approach towards optimal registration of cluster members to the head is proposed in constraint to energy gain of a cluster. The suggested approach gives a benefit of energy conservation with cluster member selection.
\end{abstract}

Key words: Multi cluster, Head selection, energy gain, wireless sensor network.

\section{INTRODUCTION}

Wireless sensor network are the upcoming approach to present need communication of physical metric in a broad area usage. Due to minimal infrastructure dependency and quicker information transmission, this network has gained and developed new reputation in engineering practice in a very short period. However, these networks are yet to be optimized to deliver the highest degree of offered service with resource conservation in WSN applications. Owing to the inconsistency in this network, resources are limited. Every node in the network has a restricted resource of bandwidth and energy supply. Due to the constraint resources the nodes are bound for minimal power dissipation to increase node lifetime which intern effects the total network lifetime of the network. Due to a dynamic deployment and routing, conservation of energy with offered service is a bottleneck task in WSN communication. Development in the area of energy conservation for WSN has outcome with several approaches for node level, topology level, or communication level energy conservation. In [1] simulated annealing approach is proposed towards energy conservation, where the approach of node deployment for energy conservation is proposed to optimize power consumption at node level. Approach such as dynamic clustering [2], operational scheduling [3,4], and interference based monitoring [5] were proposed as a suggestive approach in energy conservation in the network. A cluster alignment in energy saving based on the power distribution in the network is presented in [5]. This approach perform node placement based on energy constraint in nodes to realign the node placement in the network. In [6] scheduling approach of different phases of operation namely, sleep, wake, communicate and ideal phases were proposed in conservation energy dissipation at node level. In [7] an energy conservation approach based on the interference model of communication is proposed. This approach develops energy conservation protocol based on the interference governed at the links during communication. The energy conservation in wireless sensor network is developed as a suggestive solution for improvement of lifetime of a network. In [8] an on demand medium access control protocol (MAC) was suggested in conservation of energy in the network. The network nodes participating in communication are scheduled to operate in minimal energy dissipation and a concept of energy harvesting was presented in $[9,10]$. Harvesting approach is developed as a solution to acquire energy to a node being placed within the network. The complexity of computation in harvesting is however a developing solution. An access control approach termed RF-MAC protocol presented in [11] focusing on developing the approach of energy conservation with monitoring of access control in the network. The proposed approach uses a radio channel in improving energy level at each node level. However, the interference at channel level is unaddressed. The approach of node scheduling with distribution is outlined in $[12,13]$. The scheduling scheme conserve the energy dissipation however the exchanging of communication phases has considerable aggregated energy dissipation. At routing, mechanism in energy conservation based on power dissipation and data rate controlling is outline in [14]. This approach develops a cross layer monitoring of data exchange in energy conservation. In recent solutions, a cross layer sensing cluster routing method (ECCM) is presented in [15], where a cross layer monitoring is proposed for developing a virtual sensing layer using fog nods and optimizes the head allocation based on particle swarm optimization (PSO) for efficient energy conservation at multi cluster interface. The approach however limited to head 
placement and the participating nodes in communication and cluster formation are not observed. The node engagement in data exchange, individual energy level has a greater impact on the overall cluster energy level and intern effect the lifetime of the network. To develop an energy conservation approach in a multi cluster communication, an optimal clustering approach based on cluster energy gain is outlined in this paper. To present the stated approach, this paper is presented in 6 sections. The approach of existing EECM is outlined in section 2 . Section 3 outlines the proposed approach of energy conservation. Observed simulation results for the developed methods are outlined in section 4. Section 5 presents the conclusion for the proposed approach.

\section{ENERGY-EFFICIENT CROSS-LAYER- SENSING CLUSTERING METHOD (ECCM) [15]}

Wireless sensor network (WSN) are used as a mean of sensing and communicating different sensed data over a wireless network. The network has a great demand in practical application where the manual monitoring is difficult. This network has emerged as a promising solution in communication in different natural calamities, remote monitoring, testing, industrial and agricultural usage. The network is however a constraint of resources has such as the bandwidth and power source which limits its application in many fields. Research in energy conservation has outcome with various solutions, where node scheduling, route optimization, communication protocols, and topology based network modeling are few optimal developments. In recent past, an energy efficient cross layer sensing cluster routing method (ECCM) for energy conservation in WSN is presented in [15]. The approach focused on the development of a energy saving mechanism by an optimal selection of cluster head $(\mathrm{CH})$ based on neighbor cluster head, member nodes and the distance of nodes with cluster head, $d\left(n_{i}, \mathrm{CH}_{(p, k)}\right)$.

The EECM approach employs a Fog based network projection approach which interpolates the physical network into a fog layer of multiple cluster layers. Each of the cluster layers is developed with a cluster head and an optimization for selection of cluster head is developed using particle swarm optimization (PSO). For a transmission of data at $\mathrm{k} / \mathrm{bits}$ data rate the overall transmission energy is given by,

$$
\mathrm{E}_{\mathrm{Tx}}=\mathrm{kE}_{\text {elec }}+\mathrm{k} \varepsilon_{\mathrm{fs}} \mathrm{d}^{2}, \text { for } \mathrm{d}<\mathrm{d}_{0}
$$

Where,

$\mathrm{E}_{\text {elec }}$ is energy consumption for transmission or reception per unit of data

$\varepsilon_{\mathrm{fs}}$ is the energy consumption of transmission or reception for per unit data of unit distance in free space

$\mathrm{d}$ is distance of transmission

$\mathrm{d}_{0}$ is the range limits

The reception energy $\left(E_{R x}\right)$ is given by,

$$
\mathrm{E}_{\mathrm{Rx}}=\mathrm{kE}_{\mathrm{elec}}
$$

In the establishment of cluster PSO is employed, where the fog approach compute average energy of each node and elect possible candidate nodes. For a N node network, with $\mathrm{K}$ possible cluster and $\mathrm{M}$ candidate nodes, there exist multiple possibilities in the cluster formation. To optimize the selection, a target function is defined given as,

$$
\begin{gathered}
\mathrm{Q}_{1}=\operatorname{most}_{\mathrm{k}=1,2,3 \ldots \mathrm{K}}^{\max }\left\{\frac{\left.\sum_{\forall \mathrm{n}_{\mathrm{i}} \in \mathrm{C}_{(\mathrm{p}, \mathrm{k})} \mathrm{d}\left(\mathrm{n}_{\mathrm{i}}, \mathrm{CH}_{(\mathrm{p}, \mathrm{k})}\right)}^{\left|\mathrm{C}_{(\mathrm{p}, \mathrm{k})}\right|}\right\}}{\mathrm{f}_{2}=\frac{\sum_{\mathrm{i}=1}^{\mathrm{N}} \mathrm{E}\left(\mathrm{n}_{\mathrm{i}}\right)}{\sum_{\mathrm{k}=1}^{\mathrm{E}} \mathrm{E}\left(\mathrm{CH}_{(\mathrm{p}, \mathrm{k})}\right)}}\right.
\end{gathered}
$$

Where,

$f_{1}$ is compactness evaluation factor, given as a ratio of aggregated distance of all nodes to cluster head over number of nodes in the network.

$\mathrm{d}\left(\mathrm{n}_{\mathrm{i}}, \mathrm{CH}_{(\mathrm{p}, \mathrm{k})}\right)$ is the distance of node $\mathrm{i}$ to the cluster head $\mathrm{CH}$

$\left|\mathrm{C}_{(\mathrm{p}, \mathrm{k})}\right|$ is the number of nodes in the cluster

$\mathrm{f}_{2}$ is energy evaluation factor defined as the ratio of cluster head energy over sum of all nodes energy

$\beta$ is weight metric

In the formation of cluster, each candidate $s_{i}$ $(\mathrm{i}=1,2,3, \ldots \mathrm{K})$ broadcast a control message, containing ID, remaining energy, number of members and the distance to map fog node (MFN). Here, MFN is a virtual node developed by fog approach to communicate among cluster heads. The candidate node then computes PSO to derive optimal relaying node, derived by the convergence of a cost function,

$$
\begin{gathered}
\text { Cost }(i, j)= \\
\alpha_{1} \frac{E_{n e}\left(s_{i}\right)}{E_{c u}\left(s_{i}\right)}+\alpha_{2} \frac{N_{C H}\left(s_{j}\right)}{N_{C H}\left(s_{i}\right)}+\alpha_{3} \frac{d^{2}\left|s_{i}-s_{j}\right|+d^{2}\left|s_{j}-s_{F N}\right|}{d^{2}\left|s_{i}-s_{M F N}\right|} i \neq j
\end{gathered}
$$

$$
\operatorname{Cost}(i, j)=\alpha_{1} \frac{E_{n e}\left(s_{i}\right)}{E_{c u}\left(s_{i}\right)}+\alpha_{2} \frac{N_{C H}\left(s_{j}\right)}{N_{C H}\left(s_{i}\right)}+\alpha_{3}, i=j
$$

Where,

$\alpha_{1}, \alpha_{2}, \alpha_{3}$ are the weight coefficient which is define by ,$\alpha_{1}+\alpha_{2}+\alpha_{3}=1$

$E_{n e}\left(s_{i}\right)$ is the remaining energy for neighbor heads of $s_{i}$ $\mathrm{E}_{\mathrm{cu}}\left(\mathrm{s}_{\mathrm{i}}\right)$ is the average energy remaining in a cluster head $\mathrm{s}_{\mathrm{j}}$

$\mathrm{N}_{\mathrm{CH}}\left(\mathrm{s}_{\mathrm{i}}\right)$ is the number of member node with $\mathrm{s}_{\mathrm{i}}$

$\mathrm{N}_{\mathrm{CH}}\left(\mathrm{s}_{\mathrm{j}}\right)$ is the number of member node with $\mathrm{s}_{\mathbf{j}}$

$d\left(s_{i}, s_{j}\right)$ is the distance of cluster head $s_{i}$ to $s_{j}$

$\mathrm{d}\left(\mathrm{s}_{\mathrm{j}}, \mathrm{s}_{\mathrm{FN}}\right)$ is the distance of cluster head to fog node

$\mathrm{d}\left(\mathrm{s}_{\mathrm{j}}, \mathrm{s}_{\mathrm{MFN}}\right)$ is the distance of cluster head to map fog node

The optimization of the cost function is the minimal of the Cost $(i, j)$.

The optimal selection of cluster head is made based on the selection of probable candidates and computing the cost 
function. The convergence is made for a cluster head on satisfying minimal criteria. This optimization gives an optimal selection of cluster head and intern formation of multiple clusters which improves the communication range with energy conservation. The proposed approach has outlined the solution of cluster head selection and cluster formation. The cluster members are registered to the cluster based on the distance threshold. However, the members to a cluster head $\mathrm{NCH}$ impact the cluster performance in cluster formation and energy consumption. A derived cluster with high density of member node could dissipate energy rapidly resulting in shorter life time. Hence, a optimal cluster member registration is also needed to further improving of energy conservation. The proposed approach of member registration to a cluster head is presented in following section.

\section{ENERGY GAIN DYNAMIC CLUSTERING (EGDC)}

Selection of optimal cluster head has a significant impact on the energy saving performance in a wireless sensor network communicating in a clustered network. The presented work by EECM proposed the optimal cluster head selection in a multiple cluster approach by the cost optimization function presented in eqn. (6) and (7). The convergence achieves on the minimization of the cost function by a proper selection of head function. The cluster is formed by a cluster head and registered cluster member in the network. the cost function derived is a function of remaining energy $(\mathrm{E})$, number of nodes in the cluster $(\mathrm{N})$, and the distance value (d) given as,

$$
\cos t(i, j)=f(E, N, d)
$$

Wherein remaining energy and distance are factor of topology layout and energy saving scheme applied, number of nodes $(\mathrm{N})$ are the registered nodes in a cluster to the head selected based on distance metric. The nodes falling in the communication range limit $\left(\mathrm{d}_{0}\right)$ are registered to any of the neighboring cluster. Wherein the cost function is variant with number of nodes in the network, the registered number nodes to a head is hence has a important role in the convergence property. An optimal selection of head leads to longer lifetime, however the member density to a cluster has a direct impact on the node remaining energy (E) which is based on the communication engagement in the cluster. Hence, an optimal number of member nodes $(\mathrm{N})$ will lead to a higher energy conservation leading to longer network lifetime and faster convergence. In the optimal selection of nodes in a cluster, an energy gain based cluster formation is proposed termed as 'Energy gain dynamic clustering' (EGDC). The node selection scenario for a multi cluster network is illustrated in figure 1 below.

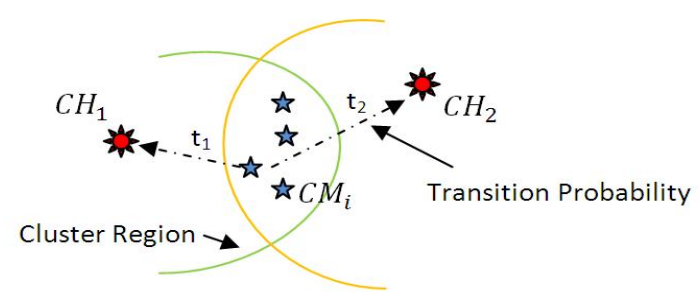

Figure 1: Member registration probability to head nodes
The transition of the member node $\mathrm{CM}_{\mathrm{i}}$ is observed to have two transitions probability $\left(\mathrm{t}_{1}, \mathrm{t}_{2}\right)$. The aggregated energy for the cluster member taking transition $t_{1}$ and $t_{2}$ is given by,

$$
\mathrm{E}_{\mathrm{a}}=\left(\sum_{\mathrm{i}=1}^{\mathrm{n}} \mathrm{E}\left(\mathrm{CM}_{\mathrm{i}}\right)\right)+\mathrm{E}\left(\mathrm{CH}_{\mathrm{p}, \mathrm{k}}\right)
$$

The optimization cost function is defined by the energy ratio of remaining energy over the aggregated energy of selected head and the number of node ratio, given by,

$$
\begin{gathered}
E_{r}=\frac{E_{n e}\left(s_{i}\right)-E_{a}}{E_{c u}\left(s_{i}\right)+E_{a}} \\
N_{a}=\frac{N_{C H}\left(s_{j}\right)+n}{N_{C H}\left(s_{i}\right)-n}
\end{gathered}
$$

The cost function of energy conservation maximization is then observed as a problem of equilibrium of number of nodes and energy ratio to minimize the cost function for convergence. The optimization to the problem addressed is governed by the energy gain factor (EGF) which is defined as a function of probability of node redundancy in engagement of communication with the energy dissipation in the network. The member nodes are selected for a cluster offering maximization of the energy gain factor. The energy gain factor is defined by,

$$
\mathrm{EGF}=\operatorname{En}\left(\mathrm{n}_{\mathrm{i}}\right)\left(\mathrm{Ea}-\mathrm{E}\left(\mathrm{Il} \mathrm{n}_{\mathrm{i}}\right)\right) \mathrm{N}_{\mathrm{a}}
$$

Where En(I) defines the probability of node engagement in data exchange and $\left(\mathrm{Ea}-\mathrm{E}\left(\mathrm{I} / \mathrm{n}_{\mathrm{i}}\right)\right)$ is the dissipation of energy for transmission and reception of information I from node $n_{i}$. The redundant probability of node engagement in information exchange is given by,

$$
\operatorname{En}\left(\mathrm{n}_{\mathrm{i}}\right)=-\sum_{\mathrm{i}=1}^{\mathrm{n}} \mathrm{P}\left(\mathrm{CH}_{\mathrm{i}}\right) \log _{2}\left(\mathrm{P}\left(\mathrm{CH}_{\mathrm{i}}\right)\right)
$$

Where $\mathrm{P}($.$) is the probability operator of data getting$ exchanged from cluster head $\mathrm{CH}_{\mathrm{i}}$. The inclusion of a member node in a cluster increases the cluster aggregate energy, however the node increase also has a impact on the communication overhead in the network. A node getting added into a cluster increases the probability of data exchange by En and hence has an overall impact on energy of $\left(E_{a}-E\right)$. The proposed approach defines the inclusion of cluster member for a maximum level of EGF. If the overall probability of network increase by $\mathrm{P}($. factor, the EGF decreases, hence a maximal point of cluster members $\left(\mathrm{N}_{\mathrm{m}}\right)$ is selected, where the EGF factor has a valley fall. This limit is set as a monitoring value of cluster member for each cluster, obtaining equilibrium of energy ratio and node ratio to converge the head selection faster. The optimal member nodes have an optimal loading probability and hence minimize the energy dissipation. This leads to longer life time in the network.

\section{SIMULATION RESULT}

The simulation evaluation of the proposed approach is analyzed with the existing approach of ECCM and conventional routing approach of Trust based secure routing (TBSR) [16]. The simulation model is defined with a randomly scatter nodes with different energy level and offered bandwidth simulated over Matlab tool. Each node is given a distinct identification (ID). The parameter 
used in the definition of the network is utilized in table 1 below.

Table 1: Simulation network parameters

\begin{tabular}{|c|c|}
\hline Network parameter & Characteristic \\
\hline Node density $\left(\mathrm{N}_{\mathrm{d}}\right)$ & 30,45 \\
\hline Network Area & $\mathrm{N}_{\mathrm{d}} \times \mathrm{N}_{\mathrm{d}}$ \\
\hline Communication range & 80 Units \\
\hline Mobility & Non-static \\
\hline Topology & Random \\
\hline MAC & 802.11 \\
\hline Power model & IEEE 802.11-NIC card \\
\hline
\end{tabular}

The network parameter is given as input to the simulation model. The network for simulation is defined with a randomly scattered nodes in a $300 \times 300 \mathrm{~m}^{2}$ network area with 30 nodes. The node communication range is set to $80 \mathrm{~m}$ range. The network is defined for multiple clusters with optimal head selection and cluster member registration. Figure 2 shows the simulated network with nodes scattered randomly, and top three selected maximum energy nodes as candidate nodes for head selection.

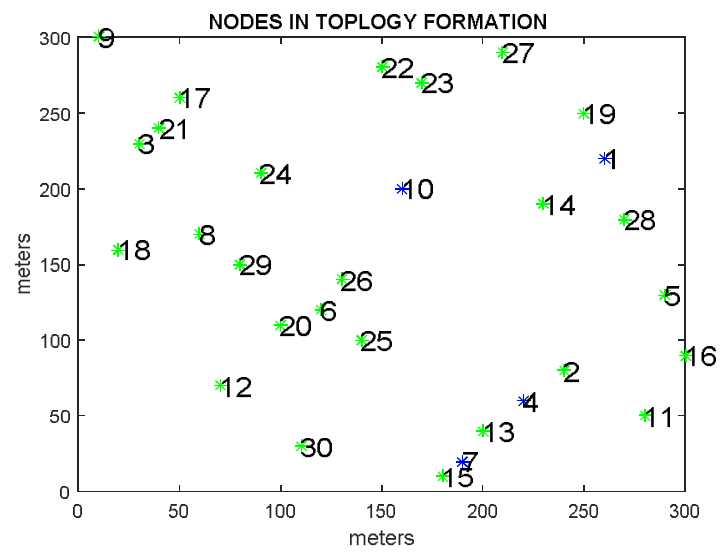

Figure 2: Simulated network with nodes and head selected

The nodes in the vicinity of the selected heads are registered to the head nodes in the range limit. However, the member nodes are added to a head based on the energy gain factor defined by the probability of data exchange in the network. Each of the cluster head is registered with nodes binding to the maximization of EGF. The communication process is developed with an extended scheduling and the routes are selected based on the residual energy model. The data packets are generated with 50 bits of information per iteration, exchanged over the simulated network. The network performance is validated with the monitoring metric of delay, network lifetime, residual energy, active number of nodes in the network, and network overhead. The observations for the simulated network with comparisons of the observing metrics are presented below.

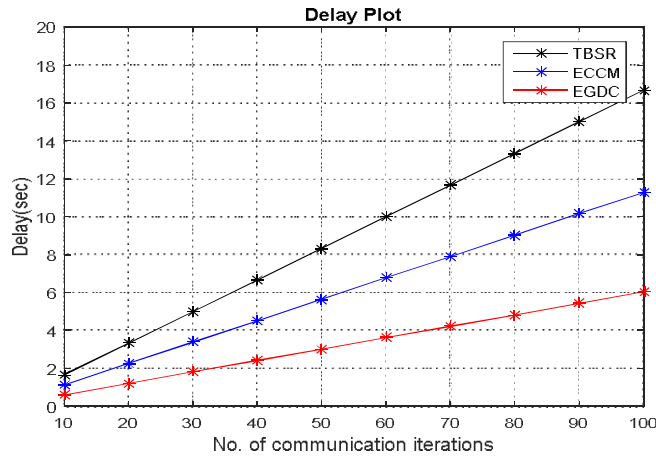

Figure 3: Delay plot for the simulated network

Delay plot for the simulation network is illustrated in figure 3. Delay is measured as the time taken for the exchange of complete data packet over the network. The proposed approach is compared with the existing approaches of ECCM and TBSR. The observation of delay plot illustrates a minimization of delay for the proposed approach compared to existing approaches. The observed delay gradually increases with increases in communication time period due to increase in the data exchange over the network and offered bandwidth. A delay in the exchange of packet is observed due to blockage built at the exchange nodes. The delay for the proposed approach is however lower due to optimal node registration leading to uniform distribution of load among different clusters.

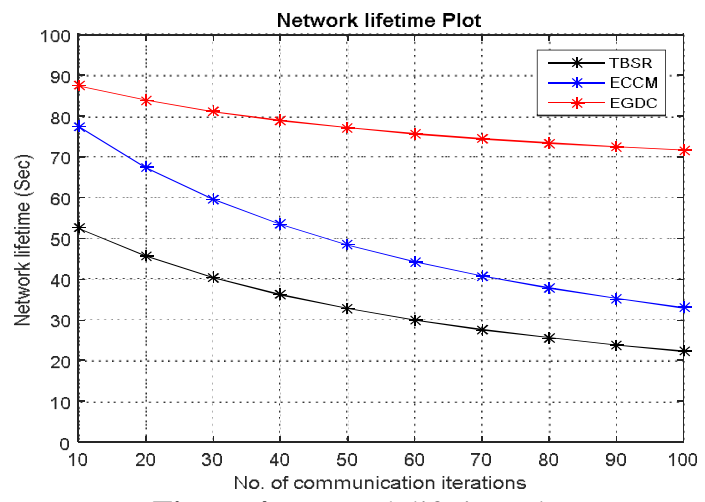

Figure 4: Network lifetime plot

The network lifetime for the network is shown in figure 4 . Network lifetime is measured as the parameter of residual energy in the network over the number of packets in exchange. The network lifetime defines the sustaining of network nodes available for data exchange. The observation illustrates an increase in network lifetime due to optimal network overhead and lower communication delay for the proposed approach, wherein the existing approaches has a random registration to cluster head leading to random load distribution which has a random energy dissipation, minimizing network life time of the network. 


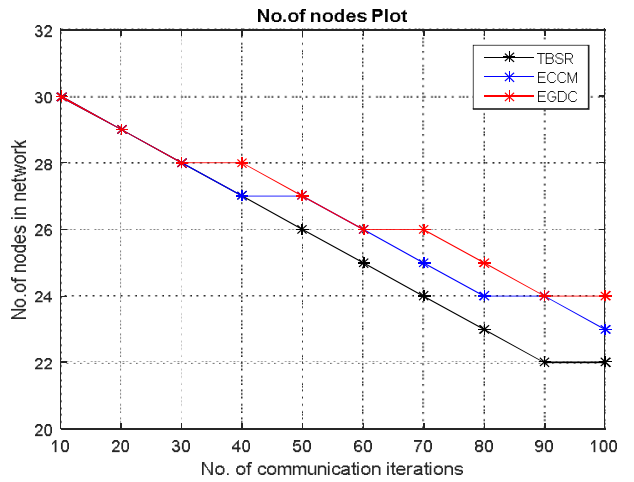

Figure 6: Comparison of Active number of nodes in the network

The number of active nodes in a network is defined as the total number of available nodes in the network with residual energy capable of communication. The active node is the count of individual nodes with available energy in the network. With process of communication, the node dissipates the transmission and reception energy and drains to minimal level. Nodes with minimal energy get eliminated resulting in active node counts lower. However, the optimal load to clusters retains the energy for a longer time in the proposed approach resulting in higher active node counts.

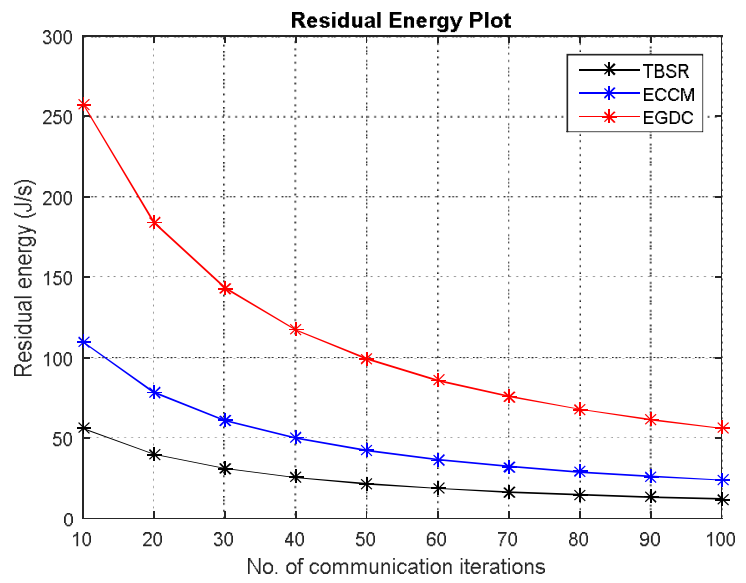

Figure 5: Residual Energy plot over communication period

\section{CONCLUSION}

The constraint of extended range and energy conservation is addressed. The selection cluster members in addition to cluster head have shown a significant performance in WSN application. Major of the developed approach has a significant usage of energy conservation in terms of head selection, however in this paper a novel approach to member registration to the cluster head is proposed. The members having probability of moving multiple clusters are optimally selected based on energy gain factor. the optimal selection of cluster member leads to uniform load distribution in the network which improves the network performance. This approach illustrated a lower delay performance, higher network life time and longer node availability in the network. The average delivery is observed to be improved with the proposed energy conservation approach.
The residual energy in the network is defined as the aggregated energy of all active nodes in the network. With increase in communication time period, the energy dissipates with each forwarding and reception of packet. An optimal head selection and monitored cluster member registration leads to higher energy conservation in the network resulting in higher residual energy.

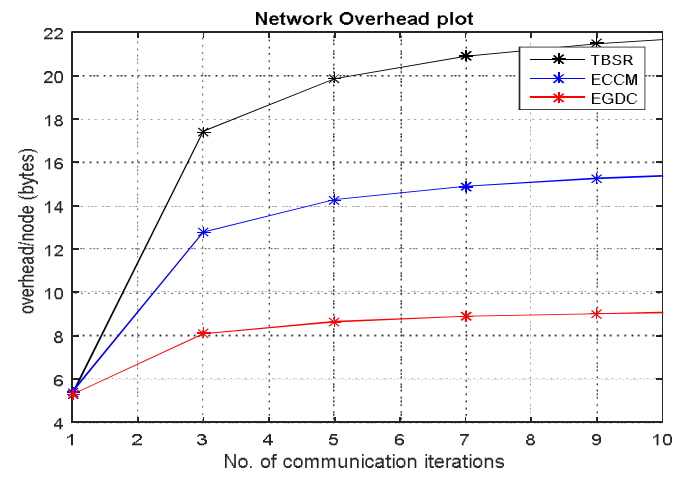

Figure 7: Overhead plot for the test network

Network overhead is the volume of data present over all nodes in the network to exchange. With more nodes retaining for a longer time the data exchange will be more, resulting in lower overhead. The lower overhead results in higher data exchange improving the data exchange probability in the network with lower energy consumption.

\section{REFERENCES}

1. Sun, Zeyu, Lili Wei, Chen Xu, Tian Wang, YalinNie, Xiaofei Xing, and Jianfeng Lu., "An energy-efficient cross-layer-sensing clustering method based on intelligent fog computing in WSNs.", IEEE Access, Vol.7, pp- 144165-144177, 2019.

2. A. Xenakis , F. Foukalas, G. Stamoulis, “ Cross-layer energy-aware topology control through Simulated Annealing for WSNs", Computers and Electrical Engineering pp-1-15, 2016.

3. Sandra Sendra, Jaime Lloret, Miguel García and José F. Toledo, "Power saving and energy optimization techniques for Wireless Sensor Networks", Journal of Communications, Sep 2011.

4. Subhash DharDwivedi, Praveen Kaushik, "Energy Efficient Routing Algorithm with sleep scheduling in Wireless Sensor Network", International Journal of 
Computer Science and Information Technologies, 2012.

5. Zilong Liao, Deshi Li, and Jian Chen, "A Handshake Based Ordered Scheduling MAC Protocol for Underwater Acoustic Local Area Networks", HINDAWI International Journal of Distributed Sensor Networks, 2015.

6. S. M. Kamruzzaman, "An Energy Efficient Multichannel MAC Protocol for Cognitive Radio Ad Hoc Networks", International Journal of Communication Networks and Information Security, Aug 2010.

7. Guodong Sun, Guofu Qiao and Lin Zhao, "Efficient link scheduling for rechargeable wireless ad hoc and sensor networks", EURASIP Journal on Wireless Communications and Networking 2013.

8. Xenofon Fafoutis, Xenofon Fafoutis, "ODMAC: An On-Demand MAC Protocol for Energy Conservation Wireless Sensor Networks", PE-WASUN'11, November, 2011.

9. ZhiAngEu, Hwee-Pink Tan, Winston K.G. Seah, "Design and performance analysis of MAC schemes for Wireless Sensor Networks Powered by Ambient Energy Conservation”, Elsevier, 6 Aug 2010.

10. Alvin C. Valera, "Survey on wakeup scheduling for environmentally-powered wireless sensor networks", Elsevier, 2014.

11. Prusayon Nintanavongsa, M. Yousof Naderi, and Kaushik R. Chowdhury, "Medium Access Control Protocol Design for Sensors Powered by Wireless Energy Transfer", IEEE, INFOCOM, 2013.

12. KeWang, Teck Yoong Chai, Wai-Choong Wong, “ Routing, power control and rate adaptation: A Qlearning-based cross-layer design", Computer Networks, Vol.102, pp-20-37, 2016.

13. Sharmila Sankar and V. Sankaranarayanan, "A Predictive Route Maintenance Protocol Based on Signal Strength for Dense Ad Hoc Networks", International Journal of Computer Theory and Engineering, August 2012.

14. A. B. M. Alim Al Islam, Chowdhury Sayeed Hyder, umayun Kabir, Mahmuda Naznin, "Stable Sensor Network (SSN): A Dynamic Clustering Technique for Maximizing Stability in Wireless Sensor Networks", scientific research wireless sensor network, 2010.

15. M.YousofNaderi, Prusayon Nintanavongsa, and Kaushik R. Chowdhury, "RF-MAC: A Medium Access Control Protocol for Re-Chargeable Sensor Networks Powered by Wireless Energy Conservation", IEEE transactions on wireless communications, July 2014.

16. J. Tang, A. Liu, J. Zhang, N. N. Xiong, Z. Zeng, and T. Wang, "A trust based secure routing scheme using the trace back approach for energy harvesting wireless sensor networks", Sensors., Vol. 18, No. 3, pp-1-43, Mar. 2018. 